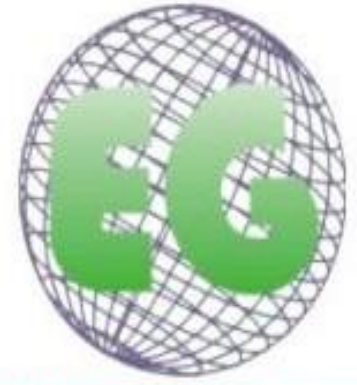

ISSN 1695-6141 $N^{\circ} 45$

\title{
Comodidad de los pacientes hospitalizados en unidades de cuidado intensivo e intermedio
}

Comfort of hospitalized patients in intermediate and intensive care units

\author{
*González Gómez, Ana **Montalvo Prieto, Amparo ***Herrera Lian, Arleth \\ *Magister en Enfermería. Docente, Universidad de Cartagena. Coordinadora de Cuidados Intensivos, \\ Clínica Medihelp. **Magister en Enfermería. Docente Universidad de Cartagena. E-mail: \\ amontalvop1@unicartagena.edu.co ***Docente, Universidad de Cartagena. Colombia.
}

http://dx.doi.org/10.6018/eglobal.16.1.260101

\section{RESUMEN}

Objetivo: Asociar los factores sociodemográficos a las dimensiones de la comodidad (físico, social, psicoespiritual y ambiental) de los pacientes hospitalizados en las Unidades de Cuidado Intensivo e Intermedio.

\begin{abstract}
Metodología: Estudio analítico transversal, realizado a 160 pacientes hospitalizados en las Unidades de Cuidado Intensivo e intermedio de cuatro instituciones de la ciudad de Cartagena, durante los meses de Julio a Septiembre del 2014. Se utilizaron dos instrumentos, la encuesta sociodemográfica y el cuestionario general de comodidad de Kolcaba. Se utilizaron la estadística descriptiva y una prueba de regresión logística para el manejo de los datos.
\end{abstract}

Resultado: El 57,5\% de los participantes fueron mujeres con 51,7 años como edad promedio, con pareja estable el $76.9 \%$, secundaria (incompleta y completa) el $43.4 \%$. Predominó en los participantes, el tipo de confort trascendencia en las dimensiones social, psicoespiritual y física y en la dimensión ambiental el tipo de confort alcanzado fue la tranquilidad. Se encontró asociación entre las dimensiones física, social y ambiental con ser de un estrato socioeconómico mayor que 2 y contar con estudios de secundaria o superiores. La dimensión psicoespiritual no presentó asociación con los factores sociodemográficos.

Conclusiones: Los pacientes hospitalizados en la $\mathrm{UCl}$ o en la UCIN con un mayor estrato socioeconómico presentaron menor probabilidad de sentir comodidad durante su hospitalización; en la dimensión social, los pacientes con mayor educación tuvieron mayor probabilidad de sentirse cómodo. El pertenecer a un estrato socioeconómico mayor que 2, reduce la probabilidad de que el paciente hospitalizado se sienta cómodo en las dimensiones ambiental y física.

Palabras clave: Cuidados intensivos; pacientes; alivio, comodidad. (Fuente: DeCS, Bireme). 


\section{ABSTRACT}

Objective: Associate the sociodemographic factors to the contexts of comfort (physical, social, psychospiritual and environmental) of hospitalized patients in the Intermediate and Intensive Care Unit.

Methodology: Analytical cross-sectional study, performed to 160 hospitalized patients in Intermediate and Intensive Care Units of four institutions in the city of Cartagena, during the months of July to September of 2014. Two instruments were used, the socio-demographic survey and the general comfort questionnaire developed by Kolcaba. Descriptive and logistic regression statistics were used for data management.

Result: $57.5 \%$ of participants were women with average age of 51.7 years, with $76.9 \%$ with a steady partner, secondary (incomplete and complete) $43.4 \%$. Transcendence was the kind of comfort that was prevailed on participants for social, psycho-spiritual and physical dimensions; for environmental dimension, tranquility was the kind of comfort achieved. It was found an association between physical, social and environmental dimensions for patients with a socioeconomic stratum higher than 2 and with secondary level educational or higher. The psycho-spiritual dimension did not showed an association with socio-demographic factors.

Conclusions: Patients hospitalized in the ICU or NICU with high socioeconomic status were less likely to feel comfort during hospitalization; in the social dimension, patients with high educational level were more likely to feel comfortable. To belong to a socioeconomic status higher than 2, reduce the likelihood that the hospitalized patient feel comfortable in the environmental and physical dimensions.

Keywords: Intensive care; patients; relief; comfort. (Source: MeSH, NLM).

\section{INTRODUCCIÓN}

La comodidad en salud hace referencia a una sensación de alivio al malestar, a un estado de tranquilidad y satisfacción, o a cualquier sentimiento que haga la vida fácil o agradable. Kolcaba (1) al definir comodidad le encontró tres sentidos (alivio, tranquilidad y trascendencia), los cuales están desarrollados en cuatro contextos (físico, psicoespiritual, ambiental y social).

En los últimos 20 años las Unidades de Cuidado Intensivo (UCI) han pasado a formar parte esencial de casi todos los hospitales generales, su importancia radica en la complejidad del tratamiento al enfermo grave y su temprana recuperación de injurias severas ${ }^{(2,3)}$, o para pacientes estables, que por su condición clínica o diagnostico necesitan ser monitorizados o de continuas intervenciones de enfermería, sin llegar a invadirlos, se hospitalizan en la unidad de Cuidado Intermedio (UCIN).

Es común que en los pacientes que se hospitalizan tanto en la UCI como en la UCIN, por presentar alguna alteración en su salud, se origina en ellos como en su familia una gran incomodidad que interrumpe su función familiar. La persona asume un papel pasivo; en muchas ocasiones, la familia queda aislada y desinformada acerca de su enfermo y no se toma en cuenta su participación en el cuidado del mismo ${ }^{(4)}$.

El paciente hospitalizado en la UCI o en la UCIN por su condición médica depende el $100 \%$ del personal de enfermería para satisfacer sus necesidades básicas. Si está consciente y orientado puede manifestar sus necesidades, deseos e incomodidades, lo que facilitaría el cuidado y mejoraría la comodidad, el paciente inconsciente, con algún grado de sedación, o con alteraciones neurológicas o mentales que se le dificulte la comunicación, al no poder manifestar sus necesidades verbalmente, enfermería debe estar atenta para satisfacerlas al igual que para interpretar cada variación en las constantes vitales, pues estas pueden en determinados momentos 
ser manifestaciones de incomodidad. Es importante brindar un cuidados de enfermería que ayuden a que los pacientes sientan comodidad durante su hospitalización.

Estar en una UCI o UCIN es una situación difícil para el paciente, porque todas las necesidades básicas (fisiológicas) deben ser satisfechas y realizadas en su unidad (cubículo) y la privacidad es muy poca por no decir nula y hacerlo en presencia de personas diferentes a su familia puede limitar al paciente para manifestar sus reales necesidades por lo que la comodidad puede alterarse. El paciente en las UCIN al estar estable y no tener medidas invasivas puede en algunos casos manifestar sus necesidades por lo que se le facilita al personal de enfermería brindar cuidado, pero al paciente se le dificulta su comodidad por alterar su privacidad.

La atención brindada a los pacientes ha evolucionado con el tiempo y estas transformaciones han llevado a que los servicios de cuidado critico en la actualidad sean considerados como un eslabón fundamental dentro de los sistemas de salud, al brindar atención al paciente en estado crítico proporcionándole además comodidad ${ }^{(4)}$, pero al brindar el cuidado de enfermería este se encuentra orientado a mejorar las condiciones de salud del paciente, sin que se tenga en cuenta su comodidad. Enfermería debe tener presente que la hospitalización de un paciente críticamente enfermo en una UCI, ocasiona un disconfort en todo el grupo familiar y la comodidad es un factor importante y relevante para el bienestar del paciente y su familia ${ }^{(4)}$.

Cuidar es la esencia de enfermería y el cuidado es el elemento paradigmático que hace la diferencia entre ésta y otras disciplinas del área de la salud ${ }^{(5)}$. El cuidado del paciente ha sido tradicionalmente visto por el personal de enfermería y el común de las personas, como propio de la profesión de enfermería. Sin embargo, el incremento en la tecnología y especializaciones en diferentes áreas específicas ha contribuido a la despersonalización del cuidado de la salud en la sociedad actual. Para mitigar esta pérdida de identidad, es fundamental realizar investigaciones que involucren los componentes del cuidado, entre estos la comodidad como indicador de su calidad ${ }^{(6)}$.

En el presente estudio las unidades que participaron forman parte de instituciones privadas, brindan cuidado a pacientes adultos pertenecientes al Sistema General de Seguridad Social, para el año 2014, presentaron un porcentaje de ocupación del $86.75 \%$ con una estancia de 5.32 días y un promedio de egreso de 28.55 pacientes por mes.

Es por esto que Kolcaba ${ }^{(4)}$ consideró que las intervenciones de enfermería debían estar encaminadas a aumentar la comodidad del paciente, dando un giro total al enfoque del cuidado biológico y pasando a un enfoque centrado en el paciente y su familia. Esta concepción da paso a hospitales humanos y cálidos donde la familia y el paciente se sientan como en su hogar y la situación de enfermedad no sea tan notoria. La comodidad de los pacientes hospitalizados en una UCl es importante para su recuperación física, emocional y espiritual, los cuidados de enfermería brindados con calidad, seguridad y humanizados favorecen el bienestar de los pacientes críticos, mejoran la repuesta a los tratamientos y su calidad de vida.

Con base en la teoría y el instrumento de Kolcaba ${ }^{(7)}$ para medir la comodidad a pacientes hospitalizados en las UCI e UCIN no se encontraron estudios, diversos estudios han identificado factores que pueden incomodar a los pacientes hospitalizados en estas unidades ${ }^{(8-10)}$. Gutiérrez ${ }^{(11)}$ en una revisión sistemática de estudios cualitativos realizada a pacientes hospitalizados en la $\mathrm{UCl}$, mostró que el 
paciente presenta miedo a la muerte cuando la siente muy cercana, desconocimiento de las consecuencias de la enfermedad y de todo lo que ocurre a su alrededor y a un futuro que cambie completamente su vida, convirtiéndole en un inválido o en una carga familiar. Otra preocupación fundamental para los pacientes es el bienestar de su familia, de su pareja y de la forma como éstos están viviendo su hospitalización.

Según Achury ${ }^{(8)}$ el paciente crítico siente que la comunicación es limitada y se convierten en un elemento emocional y situacional que influye en la experiencia del dolor porque un paciente que es incapaz de verbalizar su dolor o de hacerse comprender, les genera estados de ansiedad, agitación, depresión, tristeza y temor. De igual manera manifestó en otro estudio (9) que la presencia de factores precipitantes y predisponentes en las UCI conlleva la privación del sueño y le origina complicaciones fisiológicas, sociales y emocionales que aumentan la mortalidad y la estancia en estas unidades.

La investigadora ha observado que desde la organización administrativa y asistencial de las Instituciones Prestadoras de Salud, que en estos servicios se dispone de una infraestructura, tecnología y de apoyo científico diferente a lo dispuesto en otros servicios y para la enfermera que tiene a su cargo la dirección del cuidado en varias de las UCls de la ciudad; su preocupación principal y permanente está orientada a conservar equipos biomédicos, insumos y dispositivos, que permitan garantizar el cuidado oportuno a los pacientes hospitalizados en este servicio, lo que les permitirá la monitorización de las constantes vitales en los pacientes a su cargo, brindar un cuidado oportuno y tratar de que el paciente comprenda que las múltiples intervenciones que se le realizan, algunas pueden ser incomodas y hasta dolorosas pero mejoran y conservan su salud. Por lo anteriormente descrito, el brindar comodidad en los pacientes que se hospitalizan en UCl es una condición desconocida en este medio para el personal de enfermería y es a partir de este supuesto que nace la necesidad de conocer cómo se siente un paciente que ingresa a UCI y UCIN y poder así intervenir estas situaciones, realizando planes de cuidados que sean más intervencionista, individualizados y reales con las vivencias de los pacientes.

El fenómeno de la comodidad y la relación que se establece con las personas que necesitan asistencia en UCI y UCIN es algo positivo, que se logra como consecuencia de los cuidados que los enfermeros ofrecen y proporcionan para mejorar la situación de salud de las personas hospitalizadas ${ }^{(12)}$.

En estos pacientes el contacto con sus familiares o cuidadores es muy limitado y en algunas unidades es por pocos minutos en el día. La situación de salud del paciente hospitalizado en la UCl y la UCIN, ha sido objeto de estudio por los diferentes investigadores a nivel nacional e internacional, así como aspectos relacionados al ruido, el dolor, la conciliación del sueño del paciente hospitalizado y la comunicación del paciente en situaciones de asistencia ventilatoria invasiva, pero no se ha encontrado información acerca de la evaluación de la comodidad de los pacientes en este servicio.

Para la investigadora, el paciente hospitalizado en las UCI y la UCIN necesita además de satisfacerle las necesidades básicas por el personal de enfermería, tener medidas de comodidad las cuales deben ser brindadas de manera homogénea, con respeto, calidad, seguridad y humanización durante toda su hospitalización, esta debe ser dada en cada uno de los turnos. Se planteó como propósito del presente estudio el determinar la asociación entre los factores sociodemográficos y las dimensiones de la 
comodidad (físico, social, psicoespiritual y ambiental) presentes en los pacientes hospitalizados en las Unidades de Cuidado Intensivo e Intermedio de cuatro instituciones hospitalarias.

\section{MATERIAL Y METODOS}

Estudio analítico transversal de asociación, realizado a una muestra tomada con un nivel de confianza del $95 \%$ y un error del $5 \%$, del $50 \%$ al $70 \%$ del total de hospitalizados en un semestre en cuatro de las UCI y UCIN, para un total de 160 pacientes. El estudio se clasificó de riesgo mínimo para los participantes voluntarios, quienes debían ser mayores de 20 años ${ }^{(13,14)}$. Una vez comprendidos los objetivos y firmar el consentimiento informado -fueron conscientes de la posibilidad de retirarse y de revocarlo -, se procedió a la recolección de la información. Se obtuvo el permiso de los directores científicos de las instituciones de salud.

Se utilizó una encuesta sociodemográfica y el cuestionario general para enfermería "Comodidad en pacientes hospitalizados" diseñado y autorizado su utilización por Kolcaba ${ }^{(9)}$. Consta de 28 ítems, en una escala tipo likert de 1 a 4 . Evalúa la comodidad en 4 dimensiones: física (6 items), social (4 items), psico-espiritual (12 items), y ambiental (6 items), circunscritas estas dimensiones a los tipos de alivio, tranquilidad y trascendencia. El cuestionario se ha utilizado en pacientes oncológicos, médico-quirúrgicos, psiquiátricos y comunitarios, fue validado en Colombia y obtuvo un alfa de Cronbach de 0,9 ${ }^{(1)}$. El alfa de Cronbach obtenido en el presente estudio fue de 0,74 .

A cada paciente se le explicó cómo diligenciar los instrumentos, la información se recolectó bajo el acompañamiento de una enfermera, entrenada en su manejo. El cuestionario general de comodidad tiene un puntaje mínimo total de 28 puntos y un puntaje máximo de 112. Para el análisis estadístico se establecieron puntos de corte para cada una de la dimensiones en los tipo de alivio, tranquilidad y trascendencia, los cuales se presentan en la tabla I.

Tabla I. Distribución de los ítems y puntos de corte para cada dimensión del confort.

\begin{tabular}{|c|c|c|c|c|}
\hline Dimensión & Alivio & Tranquilidad & Trascendencia & Ítems \\
\hline Física & $0-7$ & 8-14 & $>14$ & $2,9,12,13,17.18$ \\
\hline Social & $0-5$ & $6-10$ & $>10$ & $1,3,10,22$ \\
\hline Psicoespiritual & $0-13$ & $14-26$ & $>26$ & $\begin{array}{l}4,5,6,8,15,16, \\
21,23,24,26, \\
27,28 .\end{array}$ \\
\hline Ambiental & $0-7$ & 8-14 & $>14$ & $7,11,14,19,20,25$ \\
\hline
\end{tabular}

Adaptación por los autores del instrumento Cuestionario general de comodidad de la estructura taxonómica de la teoría del confort propuesta por Kolcaba

Se utilizó un software estadístico Stata 17.0, se generaran estadísticas descriptivas sobre las características de los pacientes hospitalizados en las $\mathrm{UCl}$, utilizando frecuencias absolutas y porcentajes ${ }^{(15)}$. Para expresar la información sobre los niveles de comodidad encontrados se utilizaron medidas de frecuencia y prevalencia, 
medidas de tendencia central, varianza y desviación típica, con intervalos de confianza al $95 \%$.

\section{RESULTADOS}

El promedio de edad de los pacientes participantes en el presente estudio fue de 51,7 años, de los cuales el 57,5\% (92) pertenecían al género femenino, en cuanto al estado civil el $46.3 \%$ (74) estaba casado seguido de la unión libre en un $30.6 \%$ (49) y los solteros en un $11.9 \%$ (19). En cuanto al nivel de escolaridad de los participantes el $22.5 \%$ (36) tenía secundaria incompleta, seguido por el $21.9 \%$ (35) con secundaria completa, el 10\% (16) tiene estudios técnicos y universitarios respectivamente.

Con relación al estrato socioeconómico predominó el estrato 1 en un $41.3 \%$ (66) seguido por los estratos 2 y 3 en el $26.9 \%$ (43) y el 26,3\% (42) respectivamente. El $36.3 \%$ (58) de los pacientes participantes del estudio se encontraban afiliados en el Sistema General de Seguridad Social en Salud (SGSSS) en el régimen subsidiado y el $35 \%$ (56) de ellos al régimen contributivo. La religión predominante fue la católica, $79.4 \%$ (127) y le continuó la evangélica, 8.8\% (14) (Tabla II)

Tabla II. Distribución según sexo, estado civil, escolaridad, estrato socioeconómico y seguridad social de los pacientes hospitalizados en $\mathrm{UCl}$ y UCIN, Cartagena

\begin{tabular}{|l|c|c|}
\hline \multicolumn{1}{|c|}{ SEXO } & N & $\%$ \\
\hline Femenino ESTADO CIVIL & 92 & 57,50 \\
\hline Masculino & 68 & 42,50 \\
\hline NS/NR & $\mathbf{N}$ & $\%$ \\
\hline Casado & 3 & 1,9 \\
\hline Divorciado ESCOLARIDAD & 3 & 46,3 \\
\hline Soltero & 19 & 1,9 \\
\hline Unión libre & 49 & 11,9 \\
\hline Viudo & 12 & 30,6 \\
\hline & $\mathbf{N}$ & 7,5 \\
\hline Ninguna & 8 & $\%$ \\
\hline Primaria incomplete & 31 & 5,0 \\
\hline Primaria complete & 16 & 19,4 \\
\hline Secundaria incompleta & 36 & 10,0 \\
\hline Secundaria completa & 35 & 22,5 \\
\hline Técnico o Tecnologo & 16 & 21,9 \\
\hline Universitario & 16 & 10,0 \\
\hline Posgrado & 2 & 10,0 \\
\hline
\end{tabular}




\begin{tabular}{|l|c|c|}
\hline ESTRATO SOCIOECONOMICO & $\mathbf{N}$ & $\%$ \\
\hline 1 & 66 & 41,3 \\
\hline 2 & 43 & 26,9 \\
\hline 3 & 42 & 26,3 \\
\hline 4 & 2 & 1,3 \\
\hline 6 & 7 & 4,4 \\
\hline \multicolumn{1}{|c|}{ SEGURIDAD SOCIAL } & $\mathbf{N}$ & $\%$ \\
\hline Contributivo & 56 & 35,0 \\
\hline Regimen excepcional & 28 & 17,5 \\
\hline sin afiliación & 3 & 1,9 \\
\hline Subsidiado Total & 58 & 36,3 \\
\hline Otra & 3 & 1,9 \\
\hline NS/NR & 12 & 7,5 \\
\hline & $\mathbf{1 6 0}$ & $\mathbf{1 0 0 , 0}$ \\
\hline
\end{tabular}

Fuente: Datos del estudio.

En cuanto a los antecedentes patológicos personales y familiares, se encontró que el $44.4 \%$ (71) era hipertenso y el $14,4 \%$ (23) presentaba diabetes, en cuanto a los antecedentes de tipo familiar el 51,3\% (82) presentaba antecedentes de Hipertensión Arterial Familiar, antecedentes de Diabetes Familiar el 16.3\% (26) y problemas Cardiacos el 10.6\% (17) (Gráfico 1).

Gráfico 1. Antecedentes patológicos personales y familiares en los pacientes hospitalizados en UCN y UCIN, Cartagena.

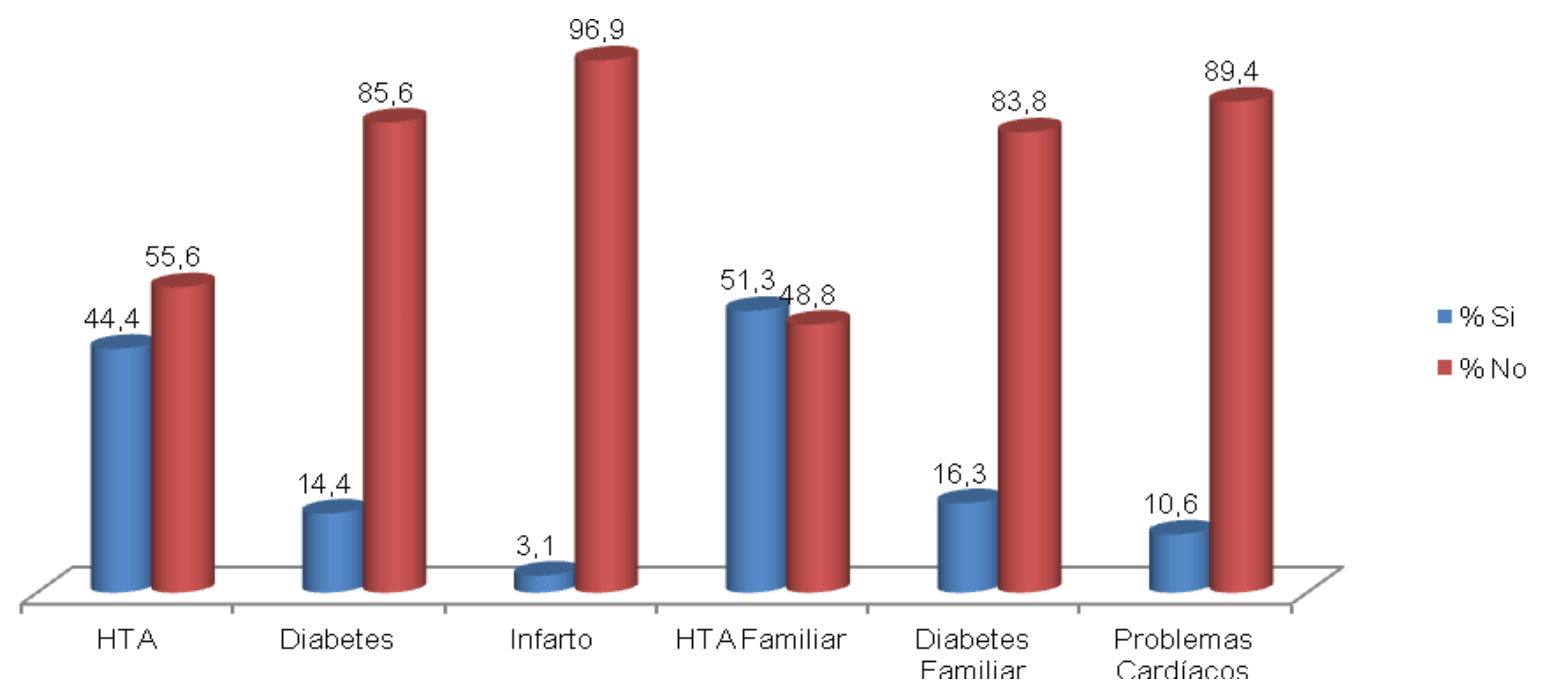

Fuente: Datos del estudio

El confort experimentado por los pacientes de acuerdo a los 3 tipos estipulados por Kolcaba (alivio, tranquilidad y trascendencia), en las dimensiones social, física, psicoespiritual en el 93,8 (150); $53.8 \%$ (86), 99.4\% (159) respectivamente. En la dimensión ambiental el tipo de confort predominante fue la tranquilidad en el $53.8 \%$ (86) (Gráfico 2). 


\section{Gráfico 2. Tipos de confort de acuerdo a cada una de las dimensiones que miden comodidad en los pacientes hospitalizados en UCl y UCIN, Cartagena.}

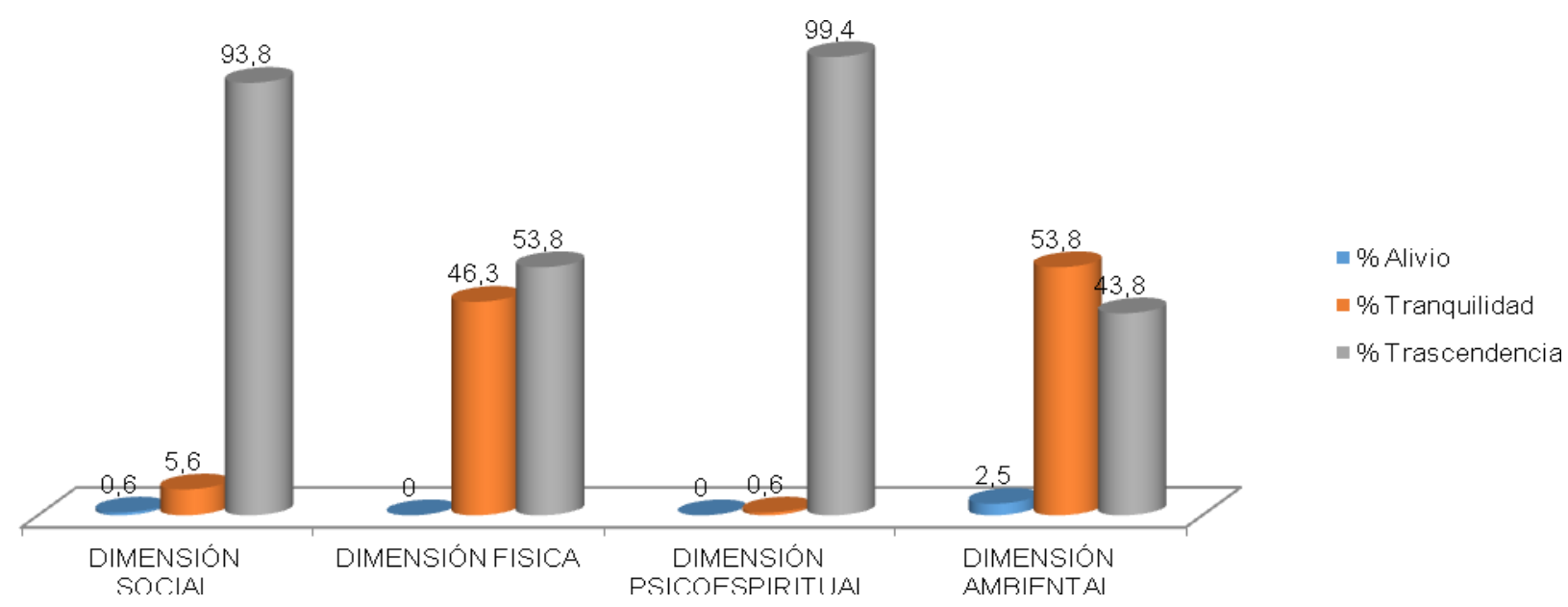

Fuente: Datos del estudio

Al establecer las asociaciones mediante la prueba de regresión logística entre los factores sociodemográficos y las dimensiones física, social, psicoespiritual y ambiental, se encontró asociación en las dimensiones física, social y ambiental. La dimensión psicoespiritual no presentó asociación con los factores sociodemográficos. En la dimensión física tener un estrato socioeconómico mayor que 2 disminuye la probabilidad de tener mayor comodidad al estar hospitalizado en una $\mathrm{UCl}$ o en la UCIN (Tabla III).

La dimensión social se asoció de forma negativa con pertenecer a un estrato socioeconómico mayor que $2(\mathrm{OR}=0,090,01-0,76)$ es decir, el pertenecer a un mayor estrato socioeconómico hay menor probabilidad de que el paciente se considere con mayor comodidad. El contar con estudios completos de secundaria 0 superiores $(\mathrm{OR}=13,991,60-122,15)$, se asocia de forma positiva puesto que a mayor educación se relaciona con mayor probabilidad de sentirse cómodo (Tabla III).

La asociación presente entre la dimensión ambiental y pertenecer a un estrato socioeconómico mayor que $2(\mathrm{OR}=0,34,0,13-0,88)$ es negativa así como el contar con estudios completos de secundaria o superiores ( $\mathrm{OR}=1,5420,668-3,563)$. Estos resultados nos muestran que para la dimensión ambiental el pertenecer a un estrato mayor que 2 y haber realizado estudios de secundaria completa o más los pacientes hospitalizados en las $\mathrm{UCl}$ o en la UCIN tienen menor probabilidad de tener mayor comodidad (Tabla III ). 
Tabla III. Regresiones logísticas de comodidad en las dimensiones física, Social y Ambiental en los pacientes hospitalizados en Unidades de Cuidado intensivo y/o intermedio, Cartagena.

\begin{tabular}{|l|c|c|c|}
\hline \multicolumn{1}{|c|}{ Variables } & $\begin{array}{c}\text { Dimensión } \\
\text { Física }\end{array}$ & Dimensión Social & $\begin{array}{c}\text { Dimensión } \\
\text { Ambiental }\end{array}$ \\
\cline { 2 - 4 } & OR I.C. 95\% & OR I.C. 95\% & OR I.C. 95\% \\
\hline Ser mujer & $1,29(0,61-2,69)$ & $3,17(0,68-14,74)$ & $0,62(0,30-1,30)$ \\
\hline $\begin{array}{l}\text { Ser de un estrato } \\
\text { mayor que 2 }\end{array}$ & $0,31(0,12-0,78)$ & $0,09(0,01-0,76)$ & $0,34(0,13-0,88)$ \\
\hline Tener pareja & $1,12(0,49-2,54)$ & $1,46(0,30-7,00)$ & $1,27(0,56-2,86)$ \\
\hline $\begin{array}{l}\text { Contar con } \\
\text { secundaria completa } \\
\text { o más }\end{array}$ & $1,06(0,46-2,41)$ & $\begin{array}{l}13,99(1,60- \\
122,15)\end{array}$ & $1,54(0,66-3,56)$ \\
\hline $\begin{array}{l}\text { Ser del régimen } \\
\text { contributivo o } \\
\text { especial }\end{array}$ & $0,60(0,27-1,30)$ & $1,05(0,17-6,38)$ & $0,68(0,32-1,46)$ \\
\hline Tener HTA & $1,44(0,66-3,10)$ & $2,54(0,48-13,33)$ & $1,16(0,55-2,46)$ \\
\hline Tener Diabetes & $1,00(0,33-3,06)$ & $1,72(0,17-17,08)$ & $0,54(0,17-1,69)$ \\
\hline Tener Infarto & $0,21(0,02-2,38)$ & $0,00(0,00-0,00)$ & $0,00(0,00-0,00)$ \\
\hline $\begin{array}{l}\text { Tener familiar } \\
\text { Hipertenso }\end{array}$ & $1,89(0,90-3,94)$ & $0,82(0,18-3,63)$ & $1,69(0,81-3,53)$ \\
\hline $\begin{array}{l}\text { Tener familiar } \\
\text { Diabetico }\end{array}$ & $1,95(0,68-5,57)$ & $0,45(0,06-3,42)$ & $1,99(0,72-5,52)$ \\
\hline $\begin{array}{l}\text { Tener familiar con } \\
\text { Problemas Cardíacos }\end{array}$ & $0,45(0,14-1,42)$ & $1,17(0,11-11,66)$ & $0,32(0,10-1,04)$ \\
\hline Fun & & & \\
\hline
\end{tabular}

Fuente: Datos del estudio

\section{DISCUSION}

Al aplicar la prueba de regresión logística a los datos obtenidos en el presente estudio y asociar las cuatro dimensiones propuestas por Kolcaba en su teoría del Confort y los factores sociodemográficos, se encontró asociación entre ser de un estrato socioeconómico mayor que 2 y contar con estudios completos de secundaria o superiores, en las dimensiones física, social y ambiental. No se presentó asociación entre la dimensión psicoespiritual y los factores sociodemográficos.

No se encontraron estudios que hicieran posible la discusión con los resultados de la presente investigación. De acuerdo a los OR obtenidos, se evidenció asociación entre el estrato socioeconómico y educación, condiciones que pueden permitirle al paciente una mejor comunicación con el personal de salud, al tiempo que expresan sus necesidades e inquietudes y aceptan los cuidados de forma más natural, valorando cada una de las actividades que les realicen. En la dimensión física, el ser de estrato socioeconómico mayor que 2, disminuye la probabilidad de sentir comodidad durante la hospitalización en la $\mathrm{UCl}$ o en la UCIN, en la dimensión social el pertenecer a un mayor estrato socioeconómico hay menor probabilidad de que el paciente sienta mayor comodidad y el contar con estudios completos de secundaria o superiores se asocia de forma positiva con la probabilidad de sentirse cómodo, para la dimensión 
ambiental los pacientes que pertenecen a un estrato mayor que 2, es menos probable que sientan una mayor comodidad en esta dimensión, aspecto que puede atribuirse a que estos pacientes viven en mejores condiciones y con más independencia económica, por lo que las limitaciones de la patología y la hospitalización pueden desagradarlos.

Con relación a las características sociodemográficas, el promedio de edad de los pacientes hospitalizados, participantes en el presente estudio fue de 51,7 años. Resultados similares encontró $\mathrm{Da}$ Silva $^{(16)}$ en un estudio de tipo descriptivo exploratorio realizado en pacientes con diagnostico principal de infarto agudo de miocardio ingresados a unidad de terapia intensiva en donde el intervalo promedio de edad estuvo entre 44 y 54 de edad; a su vez Fernandes ${ }^{(17)}$ obtuvo en su estudio, que el promedio de edad de los participantes en la investigación Estrés del paciente en $\mathrm{UCI}$, fue de 50 años; similar información encontró Marosti ${ }^{(18}$ ) en su estudio, donde el promedio de edad fue de 58,2 años. Por ser las UCI y las UCIN áreas que proporcionan atención optima al paciente con compromiso grave -real o potencial-, son los pacientes con edades superiores a la quinta década quienes ven afectadas con mayor frecuencia sus funciones vitales, y requieren atención en salud en unidades que garanticen suficientes recursos materiales y equipos de profesionales especializados (médicos y enfermeras, fundamentalmente) para poder brindar seguridad en el tipo de cuidado que recibe y garantizar su bienestar.

Con relación al estado civil, en el presente estudio el $76.9 \%$ de los pacientes tienen pareja, al respecto Da Silva ${ }^{(16)}$ reporta en su estudio que el $60 \%$ de los pacientes estaban casados, similar información presenta Marosti ${ }^{(18)}$ al reportar que el $55.8 \%$ de los pacientes estaban casados; contrario a esta información en el estudio de Fernandes ${ }^{(17)}$ solo el $25 \%$ de sus pacientes tenían pareja. Las personas que se encuentran hospitalizadas en una $\mathrm{UCI}$, su condición las hace vulnerables y el contar con una pareja, con familiares o con un grupo de amigos les permite tener un mejor apoyo social que otros pacientes que no cuentan con este recurso.

En cuanto a la escolaridad en el presente estudio el $21.9 \%$ tiene estudios de secundaria completa, al respecto, Jiménez ${ }^{(19)}$ informa en su estudio que el $29.5 \%$ tiene estudios de secundaria completa y reporta que no existe diferencia significativa entre la capacidad de afrontamiento y adaptación y la escolaridad de los familiares y pacientes que se encuentran en la UCl.

Los participantes del presente estudio pertenecían en un $53.2 \%$ a los estratos 2 y 3 , información, similar información presentó Bohórquez ${ }^{(20)}$ y Zambrano ${ }^{(21)}$ quienes reportan en sus estudios que los participantes pertenecían a estos estratos. Los pacientes que pertenecen a estos estratos están sujetos a diferentes presiones sociales y económicas que los coloca en riesgo de padecer situaciones críticas de salud que pueden requerir hospitalización en unidades de cuidado crítico, pero por sus muchas presiones de tipo socioeconómico, Bohórquez ${ }^{(20)}$ resalta en su estudio el interés que tienen los pacientes por salir de esa situación para seguir con sus actividades diarias en especial con su trabajo y de reincorporarse a su vida.

La hipertensión arterial (HTA) ocupa el primer lugar como antecedente patológico $(44.4 \%)$ y como antecedente familiar en el $51.3 \%$ de los participantes y el $16.3 \%$ de diabetes. Padrón ${ }^{(22)}$ reportó en su estudio a la HTA como el principal antecedente personal en el $60,45 \%$ de los participantes, Grillé ${ }^{(23)}$ en su estudio describe como 
factor de riesgo para el desarrollo de Hemorragia Subaracnoidea a la HTA y lo reporta como antecedente personal en el $55 \%$ de los pacientes.

A pesar de la búsqueda bibliográfica exhaustiva realizada en diferentes bases de datos y de consultar con la autora del instrumento acerca de estudios sobre comodidad en pacientes hospitalizados en las UCI e UCIN, no se encontró informes al respecto. En el presente estudio los pacientes hospitalizados en la UCI y la UCIN evaluaron el tipo de comodidad en el nivel de trascendencia en la dimensión social, en el sentido en que ellos están por encima de los problemas o el dolor propio (24), diferente calificación reportan Montalvo ${ }^{(10)}$ y Uribe ${ }^{(25)}$ en sus estudios, en donde el tipo de comodidad presentado por los pacientes fue de tranquilidad referido como un estado de satisfacción o que haga la vida fácil o agradable ${ }^{(1)}$. El tipo apoyo recibido por los pacientes de su pareja, familia y grupo de amigos ante esta condición de adversidad (estar hospitalizado en una $\mathrm{UCl}$ o en una UCIN), hace que se sientan fortalecidos y les disminuye las tensiones por encontrarse en esta situación.

Con relación a la dimensión física y psicoespiritual los datos del presente estudio reportan que el tipo de comodidad alcanzado por los pacientes fue la trascendencia, correspondiente este nivel de comodidad como al estado en que el paciente está por encima de los problemas o el dolor propio, información similar reportan los estudios de Montalvo ${ }^{(10)}$ y Gómez ${ }^{(26)}$ con relación al nivel de comodidad alcanzado por los pacientes en estas dimensiones; Uribe ${ }^{(25)}$ reporta en la dimensión psicoespiritual el nivel de trascendencia y alivio en la dimensión física referida este nivel como a la satisfacción de las necesidades específicas, lo que le permite al paciente transmitir y comunicar sus diferentes necesidades, cuando requiera cuidado.

En el presente estudio se obtuvo un nivel de tranquilidad en la dimensión ambiental, similares resultados se obtuvo en el estudio de Uribe ${ }^{(25)}$ y Montalvo ${ }^{(10)}$ referida este al estado de y satisfacción o que haga la vida fácil o agradable ${ }^{(1)}$. Dentro de la dimensión ambiental, el 59.4 \% (132) opinó que el ruido no me deja descansar, Achury ${ }^{(27)}$ expresa que los factores ambientales son el conjunto de todo lo que rodea a la persona en la UCI. Incluye, por lo tanto, los equipos biomédicos y las condiciones del servicio (iluminación o ruido), el ruido se genera por diferentes causas como las conversaciones del personal de salud (enfermería), la apertura de la puertas, las visitas de los familiares y el sonido generado por el teléfono, la radio, el televisor y los equipos de monitoreo utilizados. Estas situaciones descritas por la investigadora, coincide con los resultados del estudio, en la dimensión ambiental donde el tipo de comodidad de acuerdo a la escala de Kolcaba ${ }^{(7)}$, fue tranquilidad, los pacientes participantes no se sienten cómodos en esta dimensión y el ítems que más les genera incomodidad es el ruido.

Los datos obtenidos en las cuatro dimensiones contradicen la percepción de la investigadora acerca de la experiencia de estar hospitalizado en una UCI, por ser bastante estresante para el paciente y se considera que los resultados obtenidos pudieron deberse al número reducido de participantes, aspecto que se constituyó en una limitación del estudio. Es de anotar que una condición que debe tenerse en cuenta es que el paciente puede adaptarse fácilmente a la nueva condición que se le presenta.

\section{CONCLUSIONES}

Los pacientes participantes en el estudio fueron mujeres con edad de 51.7, católicos, con estado civil casados, con estudios de secundaria, pertenecientes al estrato 
socioeconómico 2 y afiliados al Régimen Subsidiado del Sistema de Seguridad Social de Salud.

El tipo de comodidad que predominó fue la transcendencia en las dimensiones social física, y psicoespiritual, referido este nivel a que los pacientes pueden estar por encima de los problemas, condición que puede favorecer al paciente hospitalizado en un servicio tan estresante cono UCl y UCIN.

En la dimensión ambiental el tipo de confort que predominó fue la tranquilidad lo que daría al paciente satisfacción y comodidad ante las vivencias durante su hospitalización. Dentro de la dimensión ambiental el ruido fue la mayor incomodidad reportada por los pacientes.

Para los pacientes hospitalizados en una UCI o en la UCIN en la dimensión social de la comodidad el pertenecer a un mayor estrato socioeconómico hay menor probabilidad de que se considere con mayor comodidad durante su hospitalización y tener mayor educación se relaciona con mayor probabilidad de sentirse cómodo.

Para la dimensión física de la comodidad los pacientes con un estrato socioeconómico mayor que 2 , reduce la probabilidad de tener mayor comodidad al estar hospitalizado.

En la dimensión ambiental de la comodidad los pacientes con un estrato socioeconómico mayor que 2 tienen menor probabilidad de tener mayor comodidad.

\section{RECOMENDACIONES}

Motivar al personal de enfermería como líderes en el cuidado del paciente, a realizar programas de educación sobre manejo del ruido.

Desarrollar protocolos de manejo de los equipos biomédicos -manejo de alarmas-.

Fomentar cultura del silencio, para mejorar la comodidad de los pacientes.

Realizar planes de cuidados al paciente teniendo en cuenta educación sobre la patología y los antecedentes, valorar las características del entorno y su relación con el mismo.

Realizar estudios de investigación con pacientes hospitalización en UCI y UCIN que muestren aspectos tan importantes como la comodidad que ello percibe durante la hospitalización, siendo un servicio tan especializado, no se puede apartar de la humanización en la atención, la comodidad debe ser de transcendencia en todas las dimensiones.

\section{REFERENCIAS}

(1). Dowd T. La teoría del confort. En: Marriner A, Raile M. Modelos y teorías en enfermería. 7ª . ed. Elsevier-Mosby; 2011: 706-721.

(2). Álvarez Y. Unidad de cuidados intensivos: Un campo inexplorado por el Psicólogo en Chile. [Internet] [Fecha de acceso el 30 de agosto de 2006]. Disponible en: http://csociales.uchile.cl/publicaciones/psicologia/docs/Unidad cuidadfo intensivospdf (3). Ocampo B. Apuntes para la historia del cuidado intensivo. En: Sociedad Cundinamarquesa de anestesia. SCARE. Semiología del paciente crítico, la clínica y 
la monitoria invasiva son excluyente o se complementan? 2009. (Fecha de acceso 13 de octubre de 2012). Disponible en: www.enfermeriaaps.com/portal/?wpfb dl=3872.

(4). Nava G. Estudio de caso con utilización del instrumento de Katharine Kolcaba teoría de rango medio del confort. Enf Neurol (Mex). 2010; 9(2): 94-104. (Fecha de acceso 18 de octubre de 2012). Disponible en: http://www.medigraphic.com/pdfs/enfneu/ene-2010/ene102j.pdf .

(5). Cruz M. Pérez M. Jenaro C. Flores N, Segovia MG. Necesidad Social de Formación de recurso no Profesionales para el Cuidado. Una disyuntiva para la enfermera profesional. Index de Enfermería 2010; 19(4): 269-273. (Fecha de acceso 15 de agosto de 2013). Disponible http://alfama.sim.ucm.es/wwwisis2/wwwisis.exe/[in=enflink.in]/?mfn=062111\&campo=v $300 \&$ occ $=2$.

(6). Mc Daniel A. The Caring Process in Nursing Two Instruments for Measuring Caring Behaviors. Measurement of Nursing Outcomes. En: Watson J. Assessing and Measuring Caring in Nursing and Health Science. 2a Edition, 2009: 345

(7). Kolcaba K. Taxonomic structure for the concept comfort. (Fecha de acceso 10 de diciembre de 2015). Disponible en: http://thecomfortline.com/files/pdfs/1991\%20\%20Taxonomic\%20Structure\%20of\%20Comfort\%20Theory.pdf.

(8). Achury DM. Dolor: la verdadera realidad. Rev. Aquichan. 2008; 8(2):146-158. (Fecha de acceso 13 de noviembre de 2012). Disponible en: www.redalyc.org/pdf/741/74180203.pdf

(9). Achury DM, Achury LF. Sueño en el paciente crítico: una necesidad insatisfecha en la unidad de cuidados intensivos. Rev. Investigación en Enfermería: Imagen y desarrollo. 2010; 12(1): 25-42. (Fecha de acceso 13 de noviembre de 2012). Disponible en: www.javeriana.edu.co/Facultades/Enfermeria/revista1/files/122.pdf

(10). Montalvo A., Cabrera B, Contreras J, Quiñonez S, Salem E, Serpa C. Comodidad de los pacientes hospitalizados con cáncer en dos instituciones hospitalarias, Cartagena. RFS Revista Facultad de Salud. 2014; 6(1): 20-26. (Fecha de acceso 14 de noviembre de 2015). Disponible en: http://journalusco.edu.co/index.php/RFS/article/view/608

(11). Gutiérrez B, Blanco $A$, Luque $M$, Ramírez $M$. Experiencias, percepciones y necesidades en la UCl: revisión sistemática de estudios cualitativos .Rev. Enfermería Global, 2008; 7(1): 1-4. (Fecha de acceso 15 noviembre de 2012). Disponible en: http://revistas.um.es/eglobal/article/download/822/842.

(12). Ferrer E. La medición de la comodidad en las enfermeras y pacientes oncológicos. REDUCA (Enfermería, Fisioterapia y Podología), 2009; 1(2): 501-515. (Fecha de acceso 20 de octubre de 2012). Disponible en: http://www.revistareduca.es/index.php/reduca-enfermeria/article/view/77

(13). República de Colombia. Ministerio de Salud. Resolución 008430 de 1993. (Fecha de acceso 20 de octubre de 2012). Disponible en: http://www.dib.unal.edu.co/promocion/etica res 8430 1993.pdf.

(14). República de Colombia, Ley 911 de 2004. (Fecha de acceso 14 de octubre de 2012). Disponible en; www.mineducacion.gov.co/1621/articles105034 archivo pdf.pdf

(15). Burns N, Grove S. Investigación en Enfermería. 5a. Ed. Elsevier-Saunders; 2012: 582

(16)._Da Silva S, De Oliveira MV. Respuestas humanas identificadas en pacientes con infarto agudo de miocardio ingresado en una Unidad de Terapia Intensiva. Enfermería en Cardiología 2005; 36(3): 22-27. (Fecha de acceso 12 de noviembre de 2015).

Disponible en: http://www.enfermeriaencardiologia.com/revista/3602.pdf 
(17). Fernandes L. Machado C, Fernandes, V. Salazar MB. Estrés del paciente en UCl: visión de los pacientes y del equipo de enfermería. Enfermería Global, 2013; 12(32). (Fecha de acceso 12 de noviembre de 2015). Disponible en: http://scielo.isciii.es/pdf/eg/v12n32/docencia1.pdf

(18). Marosti C, Spadoti RA. Relación entre estresores y características sociodemográficas y clínicas de pacientes internados en una unidad coronaria. Rev Latino-am enfermagem. 2006; 14(5). (Fecha de acceso 13 de noviembre de 2015) Disponible en: http://www.scielo.br/pdf/rlae/v14n5/es v14n5a12.pdf

(19). Jiménez V, Zapata L, Díaz L. Capacidad de afrontamiento y adaptación de los familiares del paciente adulto hospitalizado en una unidad de cuidado intensivo. Aquichan. 2013; 13(2): 159-172. (Fecha de acceso 13 de noviembre de 2015). Disponible http://aquichan.unisabana.edu.co/index.php/aquichan/article/view/2407/html

(20)._Bohórquez A, Ramírez A, Rodríguez E, Polentino C, Medina MC, Zureth M. Suspiro de vida: vivencias de los pacientes de la Unidad de Cuidados Intensivos sometidos a soporte ventilatorio. Revista Ciencia y Cuidado; 2011, 8(1): 61-69. (Fecha de acceso 13 de noviembre de 2015). Disponible en: http://dialnet.unirioja.es/servlet/articulo?codigo $=3853519$..

(21). Zambrano G, Ferreira J, Lindarte A, Niño C, Ramírez N, Rojas L. Entre la incertidumbre y la esperanza: Percepciones y motivaciones de los pacientes hospitalizados en la unidad de cuidados intensivos (UCl). Revista Ciencia y Cuidado; 2010, 7(1): 52-60. (Fecha de acceso 13 de noviembre de 2015). Disponible en: http://dialnet.unirioja.es/servlet/articulo? codigo $=3717275$.

(22). Padrón A., Ayala JL., Puga MS., Alonso T., Salazar T, Quiñones NA. Validación del sistema predictivo Apache II en un grupo de pacientes ingresados en la Unidad de Cuidados Intensivos. Revista Cubana de Medicina Militar, 2003; 8(2): 130-136. (Fecha de acceso 14 de noviembre de 2015). Disponible en: http://scielo.sld.cu/scielo.php?script=sci arttext\&pid=S0138-65572003000200008

(23). Grillé PM, Gallo JL, Panzardo H, Vázquez R, Bagnulo H. Hemorragia subaracnoidea en la unidad de cuidados intensivos: análisis de 97 casos clínicos. Rev Med Uruguay. 2001; 17: 114-118. (Fecha de acceso 14 de noviembre de 2015). Disponible en: www.rmu.org.uy/revista/2001v2/art5.pdf.

(24). Peterson SJ, and Bredow TS. Middle range theories: application to nursing research. Lippincott Williams \& Wilkins. 2009: 420

(25). Uribe A, Torrado I, Acevedo Y. Confort de los pacientes hospitalizados en el servicio de neurocirugía. Rev cien y cuid. 2012; 9(2): 17-25. (Fecha de acceso 13 de noviembre de 2015). Disponible en: http://webcache.googleusercontent.com/search?q=cache:FDMhb4ViPHgJ:www.imbio med.com.mx/1/1/articulos.php\%3Fmethod\%3DshowDetail\%26id articulo\%3D89334\% 26id seccion\%3D4224\%26id ejemplar\%3D8784\%26id revista\%3D260+\&cd=4\&hl=e s\&ct=clnk\&gl=co

(26). Gómez C., et al., Autopercepción de los pacientes con cáncer sobre la relación existente entre su estado nutricional, su alimentación y la enfermedad que padecen. Nutr Hosp. 2008; 23(5):477-486. (Fecha de acceso 14 de noviembre de 2015). Disponible en: http://www.redalyc.org/articulo.oa?id=309226728005

(27). Achury D, Delgado A, Ruiz M. El ruido y las actividades de enfermería: factores perturbadores del sueño. Investig. Enferm. Imagen Desarr. 2013; 15 (1): 51-63. (Fecha de acceso 12 de noviembre de 2015). Disponible en: http://revistas.javeriana.edu.co/index.php/imagenydesarrollo/article/viewFile/6025/486 $\underline{8}$ 
Recibido: 8 de abril 2016;

Aceptado: 20 de julio 2016 